\title{
Analysis of the differential urinary protein profile in IgA nephropathy patients of Uygur ethnicity
}

Zhengguang Guo ${ }^{1 \dagger}$, Zhao Wang ${ }^{1 \dagger}$, Chen $\mathrm{Lu}^{2 \dagger}$, Shufen Yang ${ }^{2}$, Haidan Sun ${ }^{1}$, Reziw ${ }^{2}$, Yu Guo ${ }^{3}$, Wei Sun ${ }^{\text {* }}$ and Hua Yue 2* $^{2^{*}}$

\begin{abstract}
Background: IgA nephropathy (IgAN) is one of the most common forms of idiopathic glomerular diseases and might lead to end-stage kidney disease. Accurate and non-invasive biomarkers for early diagnosis are required for early intervention and consequent therapy for IgAN patients. Because variance in the disease incidence and predisposing genes of IgAN has been detected among different ethnicities, the ethnicity factor should be considered in IgAN biomarker discovery. The differences in the protein profiles and pathological mechanisms of IgAN in patients of Uygur ethnicity need to be clearly illustrated.
\end{abstract}

Methods: In this study, we used urinary proteomics to discover candidate biomarkers of IgAN in patients of Uygur ethnicity. The urinary proteins from Uygur normal control and Uygur IgAN patients were extracted and analyzed using 2D-LC-MS/MS and isobaric tags for relative and absolute quantitation (iTRAQ) analysis.

Results: A total of 277 proteins were found to be differentially represented in Uygur IgAN compared with the respective normal controls. The bioinformatics analysis revealed that the immune response, cell survival, and complement system were activated in Uygur IgAN. Many differentially expressed proteins were found to be related to nephropathy and kidney injuries. Four candidate biomarkers were validated by Western blot, and these results were consistent with the iTRAQ results. ICAM1, TIMP1, SERPINC1 and ADIPOQ were upregulated in Uygur IgAN. Bioinformatic analysis revealed that the increase of ICAM1 and TIMP1 might be caused by IgAN, but the increase of SERPINC1 and ADIPOQ might be caused by proteinuria. SERPINC1 and ICAM1 were identified as the candidate biomarkers with excellent area-under-the-curve (AUC) values (0.84) for distinguishing Uygur IgAN from normal controls.

Conclusions: Using urinary proteomic analysis, we identified several candidate biomarkers for IgAN in patients of Uygur ethnicity. These results will prove helpful for exploring the pathological mechanism of IgAN in patients of Uygur ethnicity and for developing better treatments for these patients.

Keywords: IgA nephropathy, Urinary proteomics, Uygur, Biomarker

\section{Introduction}

IgA nephropathy (IgAN) is one of the most common forms of idiopathic glomerular diseases [1], and primary IgAN is characterized by the deposition of the IgA antibody in the

\footnotetext{
* Correspondence: sunwei1018@hotmail.com; yuehua3546@126.com

†Zhengguang Guo, Zhao Wang and Chen Lu contributed equally to this work.

${ }^{1}$ Core Facility of Instrument, Institute of Basic Medicine, Chinese Academy of

Medical Sciences, School of Basic Medicine, Peking Union Medical College, 5

Dong Dan San Tiao, Beijing, China

${ }^{2}$ Nephrology department, The Xinjiang Uygur Autonomous Region People's

Hospital, 91 Tianchi Road, Urumqi, Xinjiang, China

Full list of author information is available at the end of the article
}

glomerulus [2]. Overall, 25-30\% of all cases of IgAN lead to end-stage disease over a period of 20 years [3]. Early intervention and consequent therapy could prevent or delay the development of renal failure and will reduce the number of patients who require renal replacement therapy [4]. The diagnosis of IgAN requires a renal biopsy, which it is an invasive procedure with a low risk of serious bleeding complications [5]. Therefore, accurate and non-invasive biomarkers for the early diagnosis of IgAN are required.

The urinary proteome can reflect alterations in the urinary system; thus, urine is a suitable source for the discovery

(c) The Author(s). 2018 Open Access This article is distributed under the terms of the Creative Commons Attribution 4.0 International License (http://creativecommons.org/licenses/by/4.0/), which permits unrestricted use, distribution, and 
of biomarkers of kidney diseases [6]. As early as 2006, Mi-Ra Park et al. used 2-DE and MALDI-TOF MS to identify the urinary proteins showing differential expression in IgAN and normal controls and determined that 59 proteins were differentially expressed in IgAN [7]. To date, many publications have reported differentially expressed proteins in the urine, urinary exosomes and kidney tissue of IgAN patients using various proteomic methods, including MALDI-TOF [4, 7, 8], 2D-GEL [1], iTRAQ [9, 10], SILAC [11], and label-free quantification methods [12, 13]. A total of 166 candidate biomarkers have been discovered, and some of these have been validated through ELISA or Western blot analysis. The above-mentioned studies suggest that urinary proteomics could be used for the discovery of biomarkers of IgAN, and more accurate candidate urinary biomarkers for IgAN must be identified and validated.

Although IgAN has been detected among all ethnicities worldwide, it displays striking variations among different ethnicities. Specifically, IgAN is most common in Asians, moderately prevalent in Europeans, and rare in Africans [14]. Krzysztof et al. recently performed a genome-wide association study (GWAS) to identify and confirm that genome variances across a multi-ethnic cohort contribute to disease risk in different ethnicities [14]. Therefore, the ethnicity factor should be considered in the discovery of urinary biomarkers for IgAN.

Uygur is an important minority in the Xinjiang District of China, which contains over 10 million people, and the incidence of IgAN in the Uygur population is $12.5 \%[15,16]$, which is higher than common chinese population (5\%, ranged from 3 to $9 \%$ ) [17-20]. In our previous study, which was performed at the genome level, we identified five SNPs of C1GalT1 (b1,3-galactosyltransferase) [15] and $\mathrm{T}$ cell receptor alpha chain constant gene (TCRC alpha)-560 C/T polymorphism in the Uygur ethnicity [21] and assessed the relationship between genotype and the clinical presentation of IgAN in the Uygur cohort. However, the urinary proteomics of IgAN in patients of Uygur ethnicity has not been previously investigated.

In this study, the urinary proteome was investigated to discover candidate biomarkers for IgAN in the Uygur ethnicity. Urinary proteins were collected from Uygur normal controls and Uygur IgAN patients, and these two groups of pooled proteins were labeled with four-plex isobaric tags for relative and absolute quantitation (iTRAQ) reagents and analyzed by 2D-LC MS/MS. The proteins showing differential expression between IgAN patients and normal controls were functionally analyzed through Panther GO and Ingenuity Pathway Analysis (IPA). Furthermore, some select differentially expressed proteins were validated in individual samples by Western blot (Fig. 1).

\section{Materials and methods}

\section{Reagents and instruments}

HPLC-grade acetonitrile (ACN), formic acid, dithiothreitol (DTT) and iodoacetamide (IAM) were purchased from Sigma (St. Louis, MO, USA). Sequencing-grade trypsin was purchased from Promega (Madison, WI, USA). The four-plex iTRAQ reagents were purchased from ABsciex (Framingham, MA, USA). A TripleTOF 5600 mass spectrometer from ABsciex and an HPLC system from Waters (Milford, MA, USA) were used.

For the Western blot analysis, primary antibodies for adiponectin (ADIPOQ, HPA051767), intercellular adhesion molecule 1 (ICAM1, HPA002126) and antithrombin-III (SERPINC1, HPA001816) were purchased from Sigma, and primary antibodies for metalloproteinase inhibitor 1 (TIMP1, ab109125) were purchased from Abcam (Cambridge, UK).

\section{Patient selection}

In this study, 24 participants, consisting of 12 Uygur IgAN patients and 12 Uygur healthy controls, were recruited. Each group was divided into an experimental group $(n=$ $5)$ for proteomic analysis and a validation group $(n=7)$ for Western blot validation. The clinical characteristics of all of the participants are shown in Table 1 (detailed

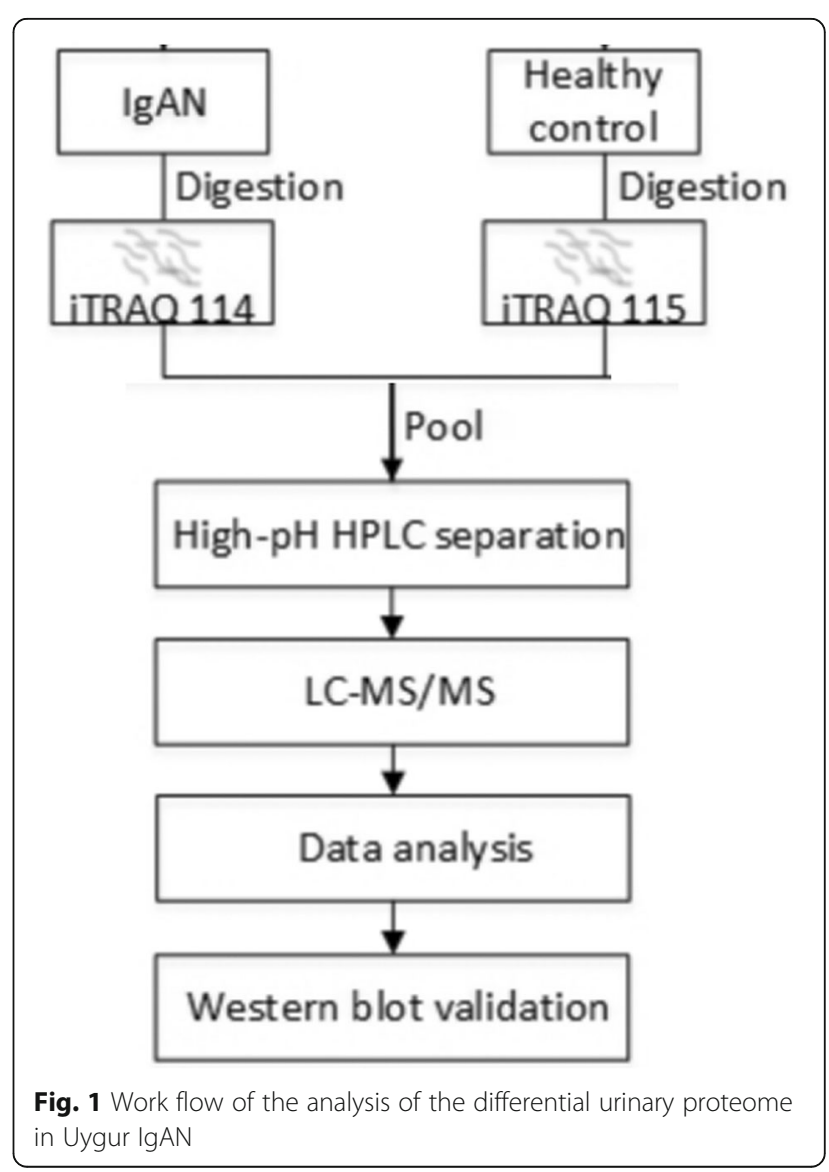


information is provided in Additional file 1: Table S1). The age, systolic blood pressure (SBP), diastolic blood pressure (DBP), body mass index (BMI), serum creatinine (SCr), blood urea nitrogen (BUN), urinary albumin excretion rates (UAER), estimated glomerular filtration rate (eGFR), triglycerides (TG), serum albumin (Alb), lowdensity lipoprotein (LDL), high-density lipoprotein (HDL) and fasting blood glucose (FBG) in the two groups were measured. TThis study was approved by the Institutional Review Board of the Institute of Basic Medical Sciences and conformed to the principles outlined in the Declaration of Helsinki. Committee's reference number: 0362014. Because our study used the remaining waste samples of the participants from clinical examination, and did not directly contact with the participants and would not regarded privacy of the participants, the Institutional Review Board of the Institute of Basic Medical Sciences waived the informed consents.

\section{Urinary protein extraction, protein digestion, and ITRAQ labeling}

The urinary protein extraction, protein digestion and iTRAQ labeling protocols used in this study were described in our previous publication [6]. In detail, urinary samples collected in the morning from the two groups were centrifuged at $5000 \mathrm{~g}$ for $30 \mathrm{~min}$ to remove the precipitates. The supernatants were precipitated by three volumes of ethanol and re-suspended in lysis buffer (containing $7 \mathrm{M}$ urea, $2 \mathrm{M}$ thiourea, $0.1 \mathrm{M}$ DTT and 50 $\mathrm{mM}$ Tris). The protein concentration of each sample was estimated using the Bradford method. Equal amounts of normalized total protein were taken from each of the donors in the experimental groups $(n=5$ in each group), and $200 \mu \mathrm{g}$ of protein from each sample within each group was pooled.

The filter-aided sample preparation (FASP) method was used for protein digestion with trypsin. First, $1 \mathrm{mg}$ of the pooled proteins in the control and patient groups was reduced by $20 \mathrm{mM}$ DTT at $37^{\circ} \mathrm{C}$ for $1 \mathrm{~h}$ and alkylated by $50 \mathrm{mM}$ IAM at room temperature in the dark for $45 \mathrm{~min}$. The samples were then loaded onto a 10-kDa ultrafilter tube (Pall, Port Washington, NY, USA) and washed twice with $8 \mathrm{M}$ urea and then twice with $25 \mathrm{mM} \mathrm{NH}_{4} \mathrm{HCO}_{3}$. After trypsin dissolved in 25 $\mathrm{mM} \mathrm{NH} \mathrm{NHCO}_{3}$ was added to the protein samples, the samples were digested at $37{ }^{\circ} \mathrm{C}$ overnight. The digested peptides were collected by filtration and then subjected to desalting using an Oasis C18 column. The 100- $\mu \mathrm{g}$ peptide in the IgAN and control samples was individually labeled with the 114 and 115 four-plex iTRAQ reagents according to the manufacturer's protocol (ABsciex). We used equal amounts of total protein for sample normalization. Finally, the samples were pooled for 2D-LC-MS/MS analysis.

\section{D LC-MS/MS analysis}

The pooled iTRAQ labeled samples were first fractionated using a high-pH RPLC column from Waters (4.6 $\mathrm{mm} \times 250 \mathrm{~mm}$, Xbridge C18, $3 \mu \mathrm{m})$. A mass of $200 \mu \mathrm{g}$ of the pooled iTRAQ-labeled samples was loaded onto the column in buffer A1 $(0.1 \%$ aqueous ammonia in water, $\mathrm{pH} 10)$ and eluted with buffer B1 (0.1\% aqueous ammonia in $10 \%$ water and $90 \% \mathrm{ACN}, \mathrm{pH} 10$, flow rate $=0.6$ $\mathrm{mL} / \mathrm{min}$ ) with a gradient ranging from 5 to $25 \%$ for 60

Table 1 Clinical characteristic of Uygur normal controls and IgAN patients

\begin{tabular}{lll}
\hline Characteristics & Uygur normal controls $(n=12)$ & Uygur IgAN patients $(n=12)$ \\
\hline Gender $(\mathrm{M} / \mathrm{F})$ & $9 / 3$ & $6 / 6$ \\
Age $($ year $)$ & $43.8 \pm 8.1$ & $38.7 \pm 8.3$ \\
BMI $(\mathrm{mg} / \mathrm{m} 2)$ & $21.1 \pm 1.7$ & $24.3 \pm 2.8^{\mathrm{b}}$ \\
$\mathrm{SCr}(\mu \mathrm{mol} / \mathrm{L})$ & $86.9 \pm 9.7$ & $166.2 \pm 182.1$ \\
BUN $(\mathrm{mmol} / \mathrm{L})$ & $5.9 \pm 1.2$ & $9.1 \pm 5.9^{\mathrm{a}}$ \\
UAE $(\mu \mathrm{g} / \mathrm{min})$ & $11.8 \pm 1.8$ & $349.8 \pm 115.1^{\mathrm{b}}$ \\
eGFR $(\mathrm{m} / \mathrm{min})$ & $97.9 \pm 7$ & $65.4 \pm 34.2^{\mathrm{b}}$ \\
Alb $(\mathrm{g} / \mathrm{l})$ & $48.9 \pm 4.5$ & $38.6 \pm 4.9^{\mathrm{b}}$ \\
TG $(\mathrm{mmol} / \mathrm{L})$ & $1.3 \pm 0.4$ & $1.7 \pm 0.9$ \\
SBP $(\mathrm{mmHg})$ & $128.1 \pm 10.3$ & $132.7 \pm 17.9$ \\
DBP $(\mathrm{mmHg})$ & $80.2 \pm 5.3$ & $83.3 \pm 11.1$ \\
LDL $(\mathrm{mmo} / \mathrm{L})$ & $2.6 \pm 0.4$ & $2.7 \pm 0.9$ \\
HDL $(\mathrm{mmol} / \mathrm{L})$ & $1.5 \pm 0.4$ & $1.2 \pm 0.3$ \\
FBG $(\mathrm{mmol} / \mathrm{L})$ & $4.6 \pm 0.3$ & $4.5 \pm 0.8$ \\
\hline
\end{tabular}

$B M I$ body mass index, $S C r$ serum creatinine, BUN blood urea nitrogen, UAE urine albumin, eGFR estimated glomerular filtration rate, Alb serum albumin, TG triglyceride, SBP systolic blood pressure, DBP diastolic blood pressure, $L D L$ low-density lipoprotein, $H D L$ high-density lipoprotein, $F B G$ fasting blood glucose ${ }^{a} p<0.05$ for IgAN versus its respective normal control; ${ }^{b} p<0.001$ for IgAN versus its respective normal control 
min. The eluted peptides were collected as 60 fractions, with one fraction collected per minute, and were pooled to obtain 20 samples. Each fraction was analyzed with a reverse-phase-C18 self-packed capillary LC column $(75 \mu \mathrm{m} \times 100 \mathrm{~mm})$. A TripleTOF 5600 mass spectrometer was used for the LC-MS analysis. The MS data were acquired under the conditions described in our previous publication [6]. Each fraction was run twice.

\section{Data processing}

Mascot software (Matrix Science, London, UK; version 2.5.01) was used for the database searching of all of the samples. In Mascot, the database was set to the Swiss-Prot human database, and the digestion enzyme was set to trypsin. The parent ion mass tolerance was $0.02 \mathrm{Da}$, and the fragment ion was $0.05 \mathrm{Da}$. Carbamidomethyl of cysteine and the four-plex iTRAQ labeling were set as fixed modifications, and a maximum of two miscleavage sites was allowed. For protein identification, Scaffold (version Scaffold_4.0.7, Proteome Software Inc., Portland, OR, USA) was used. During the process of protein assembly, some shared peptides were assigned to a group of proteins but could not be assigned to a specific protein. These groups of proteins that were identified based on these shared peptides were clustered as a protein group. To acquire the confidential identification results, peptide and protein identification was set to a FDR of less than $1.0 \%$ on both the peptide and protein levels, and a protein group with at least one unique peptide that was not shared with other protein groups was set as the filtering criteria. To meet the principle of simplicity, Scaffold Q+ (version Scaffold_4.3.2, Proteome Software Inc., Portland, OR, USA) was used for the iTRAQ quantification. The acquired intensities in the experiment were normalized globally in all runs. The healthy controls were set as the reference channels, which were normalized to produce a 1:1-fold change, resulting in all of the proteins from the healthy controls having a value of 1 . The reporter ion peak intensity below $1 \%$ of the highest peak intensity in a spectrum was unreliable for quantification and removed from the quantification [6].

To estimate the relative abundance of proteins in the urine of IgAN, the acquired .wiff files were imported into Progenesis software (Nonlinear Dynamics, Version 4.1) for label-free quantification analysis. The features were filtered for charge state $(+2$ to +5$)$. The MS/MS spectra from all runs were exported and searched against the SwissProt human database using Mascot with the same parameters above. The results were imported back into Progenesis, and the peptide identifications were mapped onto the quantitative peptide data. The protein abundance was summarized from all quantification from the 20 runs. The relative abundance of one protein is calculated by the abundance of one protein/ the abundance of all proteins in urine.

\section{GO and IPA analysis}

For the GO analysis, all of the differential proteins were analyzed using the Panther database (http:// www.pantherdb.org/) and compared with urinary database. The proteins were classified based on their molecular function, biological process and cellular component categories in the Gene Ontology (GO) annotations.

For the IPA analysis, the differential proteins were analyzed using IPA software (Ingenuity Systems, Mountain View, CA, USA). The proteins were mapped to the IPA database and other databases in the disease and the functional categories and canonical pathways categories with $\mathrm{Z}$-score and $P$-value rankings, respectively.

\section{Western blot analysis}

Four selected candidate biomarkers, namely ADIPOQ, SERPINC1, ICAM1, and TIMP1, were validated in individual samples from the experimental and validation groups by Western blot. In detail, the protein lysates were separated on a $6 \%$ SDS-polyacrylamide gel, electrotransferred to polyvinylidene fluoride (PVDF, Immobilon P, Millipore) membranes, and blocked using 5\% nonfat dry milk in Tris-buffered saline at pH 7.5 (TBST, 100 $\mathrm{mmol} / \mathrm{L} \mathrm{NaCl}, 50 \mathrm{mmol} / \mathrm{L}$ Tris, and $0.1 \%$ Tween-20). The membranes were immunoblotted with primary antibodies against the candidate proteins and then with secondary antibodies that were conjugated to horseradish peroxidase (HRP) [6]. The chemiluminescence signals were collected using an LAS 4000 system (ImageQuant LAS 4000 mini, General Electric Company, Boston, MA, USA), and the subsequent quantitative analysis was performed using Image software. The ROC curves were drawn using SPSS 18.0.

\section{Results \\ Qualitative and quantitative analysis of the urinary proteome}

IgAN patients and normal controls were included in this study. The UAER, SCr and BUN levels were increased by 28 -fold, 91 and 54\%, respectively, and the eGFR and serum Alb levels were decreased significantly by 33 and $21 \%$, respectively, in the Uygur IgAN group compared with the Uygur normal controls. Additionally, the BMI index was increased in the Uygur IgAN patients. Other indexes showed no significant differences between the two groups (Table 1, detailed data are shown in Additional file 1: Table S1).

The urinary proteins from the normal control experimental group $(n=5)$ and the IgAN experimental group $(n=5)$ were extracted, and the samples from each 
group were pooled. Each of the pooled samples was subjected to iTRAQ labeling and analyzed by 2DLCMS/MS. A search of the human Swiss-Prot database using the Mascot algorithm with a 1\% false discovery rate (FDR) at both the peptide and protein levels identified a total of 1025 protein groups with at least one peptide. Specifically, a total of $911 / 877$ proteins and $2724 / 2697$ peptides were identified in the first/second run of the fractions (detailed data are shown in Additional file 2: Table S2). Using a ratio-fold change greater than 2, 277 proteins were found to be differentially represented in Uygur IgAN patients compared with the normal controls, and of these 233 and 44 were upregulated and downregulated, respectively (detailed data are shown in Additional file 3: Table S3).

\section{GO and IPA analyses}

To further study the biological function of the proteins showing differential expression between Uygur IgAN patients and the normal controls, the 277 differentially expressed proteins were subjected to GO and IPA analyses.

The proteins showing differential expression in Uygur IgAN were searched for the enrichment of GO terms in the Panther Classification System with the urinary database. The differentially expressed proteins were classified into the molecular function, biological process and cellular component categorizes (Fig. 2). In the molecular function category, antioxidant activity was overrepresented in IgAN ( $p$-value $=1.0 \mathrm{E}-08$, enrichment score $=$ 16). In the biological process category, the immune response process were overrepresented ( $p$-value $=5.0 \mathrm{E}-11$, enrichment score $=3.7)$. In the cellular component category, extracellular proteins $(p$-value $=3.2 \mathrm{E}-43$, enrichment score $=3.6)$ and extracellular matrix proteins $(p$-value $=1.7 \mathrm{E}-04$, enrichment score $=2.4)$ were overrepresented, whereas intracellular proteins were underrepresented in IgAN. Several extracellular matrix-related proteins, including MMPs and TIMPs, showed differential expression in the urine of IgAN patients, indicating that the deposition of extracellular proteins was increased in the mesangium in IgAN.

To further analyze the detailed functional changes in Uygur IgAN, an IPA analysis was performed. The disease and biofunction analysis revealed that the immune response and fatty acid metabolism were activated in IgAN. Additionally, cell viability and survival were activated in IgAN, which might reflect the compensatory response of the kidney to resist kidney damage caused by IgA nephropathy (Fig. 3a).

To detail the detection of the molecular mechanism of IgAN, a canonical pathway analysis was performed. For example, the immune response related pathway were activated in IgAN, which indicates that the immune system is activated in IgA nephropathy [22, 23]. The complement system was also found to be activated in Uygur IgAN, which demonstrates that local complement activation plays a critical role in glomerular injury in IgAN [22] By IPA analysis, we also found that 16 differential proteins associated with nephropathies or kidney dysfunctions. These proteins might be candidate biomarkers of IgAN (Fig. 3c).

Therefore, the urinary proteome could provide comprehensive pathological and physiological information of IgAN in patients of Uygur ethnicity.

\section{Western blot validation}

By IPA analysis, four proteins, including ICAM1, TIMP1, ADIPOQ and SERPINC1 were functional related to IgAN or kidney injury in previous studies. SERPINC1 is associated with dysfunction of the kidney and chronic renal failure [10, 24]. ADIPOQ is associated with chronic kidney failure, end-stage renal disease, and renal hypertrophy [25-27]. TIMP1 play a role in the adhesion of kidney cells [28, 29]. ICAM1 is associated with proteinuria, renal tubule injury and kidney injury [30-34]. In addition, ADIPOQ and SERPINC1 have reported to be upregulated in the urine and plasma of IgAN in previous studies [10, 24, 35]. These four proteins were selected for western blot validation. (The evidences of the four proteins on IgAN were shown in Table 2). All the four proteins were validated in both the experimental group $(n=5)$ and the validation group $(n=7)$ of the IgAN patients (total $n=12$ ) and normal controls (total $n=12$ ).

As shown in Table 3, the Western blot results for all four differentially expressed proteins revealed similar trends to those found through the iTRAQ analysis. SERPINC1, ADIPOQ, ICAM1, and TIMP1 were all upregulated in the Uygur IgAN patients (Fig 4a-d and Table 3).

To evaluate the diagnostic effects of the four candidate biomarkers, which were found to be upregulated in Uygur IgAN, the ROC curves were plotted. As shown in Fig. 4a and $\mathrm{d}$, ADIPOQ and TIMP1 showed moderate sensitivity and specificity for the diagnosis of IgAN, with area-under-the-curve (AUC) values of 0.701 and 0.639, respectively. In contrast, SERPINC1 (Fig. 4b) and ICAM1 (Fig. 4c) showed excellent sensitivity and specificity for the diagnosis of IgAN, with AUC values of 0.840 and 0.840 , respectively, indicating that SERPINC1 and ICAM1 are potential biomarkers for the diagnosis of IgAN in the Uygur ethnicity. In addition, missing protein bands for the four proteins were observed in some cases, particularly in the normal control group, and this finding might be due to the fact that the amounts of the four proteins in some normal control cases were lower than the limit of detection by Western blot. This low amount of the four proteins in some cases 


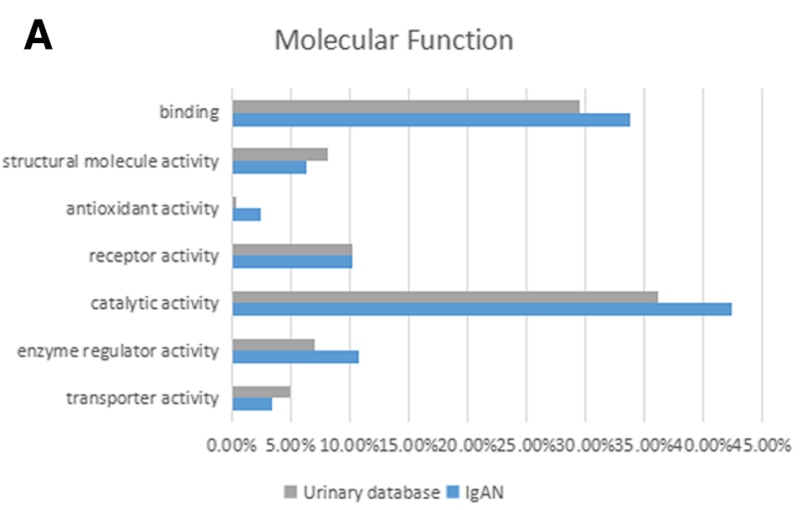

B Biological Process

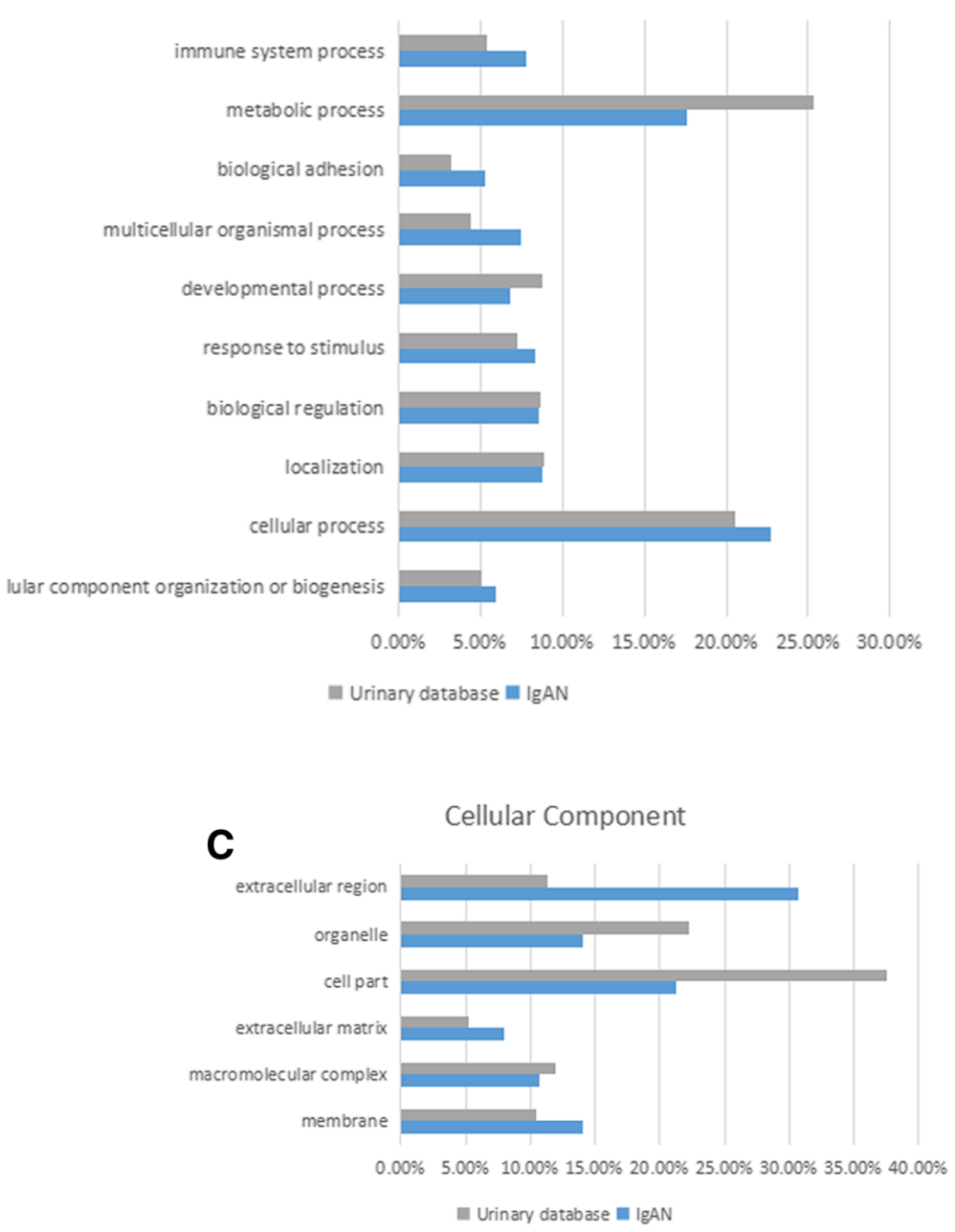

Fig. 2 GO analysis of differentially expressed proteins in Uygur IgAN. Differentially expressed proteins in Uygur IgAN were classified into the molecular function (a), biological process (b), and cellular component (c) categories of human genes compared with the entire human normal urinary proteome through GO analysis. Categories with a constitution of at least $2 \%$ are displayed in the bar charts

might be caused by inter-individual variations. Previous studies have shown inter-individual variations in urinary proteins of $20-60 \%$ in normal humans [36,37], and higher inter-individual variations might exist in IgAN patients.

\section{Discussion}

In this study, we used a normal Uygur control group and IgAN patients of Uygur ethnicity to evaluate the characteristics of IgAN urinary proteomics in a Uygur 


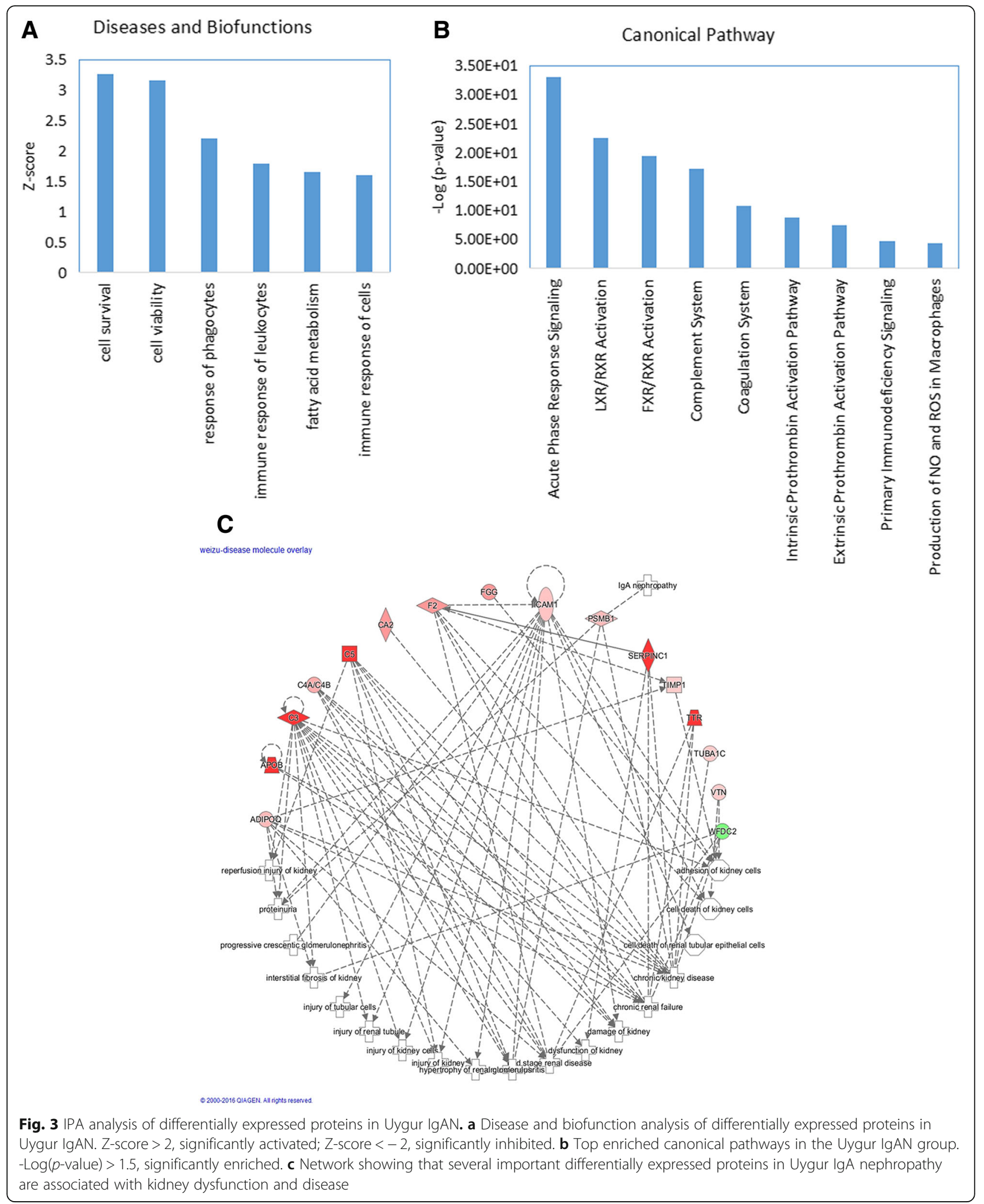

cohort. A total of 277 proteins showed differential expression in the Uygur IgAN samples. By IPA analysis, we found that some of the differential proteins in IgAN like lipid and coagulation proteins were found abundantly in serum and thus likely serum-leaked into urine. To detect whether the four IgAN potential biomarkers were leaked 
Table 2 The evidence of the four differential proteins on IgAN or kidney injury in tissue level, body fluid level and functional level

\begin{tabular}{|c|c|c|c|c|c|}
\hline $\begin{array}{l}\text { Protein } \\
\text { name }\end{array}$ & $\begin{array}{l}\text { Kidney tissue } \\
\text { evidence on IgAN }\end{array}$ & $\begin{array}{l}\text { Kidney tissue evidence } \\
\text { on IgAN method }\end{array}$ & $\begin{array}{l}\text { Body floid } \\
\text { evidence } \\
\text { on IgAN }\end{array}$ & $\begin{array}{l}\text { Body floid evidence } \\
\text { on IgAN method }\end{array}$ & $\begin{array}{l}\text { Evidence in kidney injury and other } \\
\text { kidney diease }\end{array}$ \\
\hline ADIPOQ & & & $\begin{array}{l}\text { serum } \uparrow \\
\text { urinary } \uparrow[35]\end{array}$ & $\begin{array}{l}\text { radio-immunity } \\
\text { analysis }\end{array}$ & $\begin{array}{l}\text { 1. an adipocyte-specific plasma protein } \\
\text { 2. accumulates in the injured kidney, } \\
\text { modulating inflammation and oxidative } \\
\text { stress. [25, 26] } \\
\text { 3. induces kidney apoptosis in Ischemia- } \\
\text { reperfusion injury [27]. }\end{array}$ \\
\hline SERPINC1 & $\begin{array}{l}\text { capillary walls of } \\
\text { the glomeruli [24] }\end{array}$ & immunofluorescence & $\begin{array}{l}\text { urinary } \uparrow \\
{[10,24]}\end{array}$ & $\begin{array}{l}\text { Laurell rocket } \\
\text { immunoelectrophoresis; } \\
\text { iTRAQ quantification }\end{array}$ & $\begin{array}{l}\text { 1. Serine protease inhibitors in plasma } \\
\text { that inhibits the blood coagulation } \\
\text { cascade. }\end{array}$ \\
\hline ICAM1 & $\begin{array}{l}\text { The tubular and } \\
\text { interstitial } \\
\text { expression [30] }\end{array}$ & $\begin{array}{l}\text { avidin-biotin- } \\
\text { peroxidase }\end{array}$ & & & $\begin{array}{l}\text { 1. a member of ligands for the leukocyte } \\
\text { adhesion protein LFA-1 (integrin alpha- } \\
\text { L/beta-2). } \\
\text { 2. Increases in acute renal allograft rejection } \\
\text { [31], chronic kidney disease (CKD) [32] } \\
\text { and lupus nephritis }[33,34] \text { in urine. }\end{array}$ \\
\hline TIMP1 & & & & & $\begin{array}{l}\text { 1. A positive regulator of ECMs in the } \\
\text { mesangium. } \\
\text { 2. Elevated in acute kidney injury (AKI) after } \\
\text { liver transplantation [28] } \\
\text { 3. Overexpressed in the kidney tissue of } \\
\text { diabetic nephropathy rats [29]. }\end{array}$ \\
\hline
\end{tabular}

from serum or not, we compared the relative abundance of the four proteins in urine of IgAN, and in the serum (data from Geyer's study [38]). The serum protein data were generated from quantitative label-free LC-MS/MS analysis of normal human serum and the protein abundance were evaluated using peptide intensity [38]. We also analyzed the IgAN differential protein abundance in urine using the same method. If the relative abundance of one protein in the urine is less than or equal to that in serum, it was considered as serum leaked protein; If the relative abundance of one protein in the urine is greater than that in serum, it might be a urinary enriched protein, which could be considered as IgAN related protein. The relative abundance of TIMP1 and ICAM1 in the urine of IgAN was higher than those in the serum. These two proteins were enriched in the urine, indicating TIMP1 and ICAM1 were not simply leaked from the serum, but generated from the IgAN. The relative abundance of SERPINC1 and ADIPOQ in the urine of IgAN was lower than those in the serum, thus, serum-leakage could not be completely eliminated. (Additional file 4: Table S4).
In the glomerulonephropathy, the serum proteins might be leaked into the urine, and led to proteinuria. To further confirm whether the increase of 4 proteins is caused by serum-leakage or by IgAN, we compared the changes of four proteins in the urine of IgAN and other glomerulonephropathy (microalbuminuria DN). If one protein is up-regulated in both IgAN and microalbuminuria $\mathrm{DN}$, it might be caused by serumleakage; if one protein is only up-regulated in IgAN, it might be caused by IgAN itself. Guo et al. [6] compared the urinary $\mathrm{N}$-glycoproteins in normal control, DN normalbuminuria, DN microalbuminuira and DN macroalbuminuria. ICAM1 and TIMP1 were not increased in the urine of microalbuminuria compared to the normalbuminuria, indicating these two proteins were not serum leaked proteins. While the SERPINC1 increase in the urine of microalbuminuria and macroalbuminuria [6]. ADIPOQ was O-glycoprotein, and was not identified in Guo's study. In another study [35], ADIPOQ was up-regulated in the urine of DN (macroalbuminuria) compared to healthy control. These results indicated that SERPINC1 and ADIPOQ

Table 3 Quantitative results from the iTRAQ and Western blot analysis of four differentially expressed urinary proteins

\begin{tabular}{lllll}
\hline Gene Name & Accession number & iTRAQ quantification & Western blot & AUC value \\
\hline Adiponectin (ADIPOQ) & Q15848 & $1: 3.94$ & $1: 1.87$ & 0.701 \\
Antithrombin-III (SERPINC1) & P01008 & $1: 13.86$ & $1: 2.82^{*}$ & 0.840 \\
Intercellular adhesion molecule 1 (ICAM1) & P05362 & $1: 2.06$ & $1: 2.77^{*}$ & 0.840 \\
Metalloproteinase inhibitor 1 (TIMP1) & P01033 & $1: 3.25$ & $1: 5.89^{*}$ & 0.639 \\
\hline
\end{tabular}

(Normal control: IgAN). The AUC values of the ROCs of the four candidate biomarkers are shown ${ }^{*} p<0.05$ 
A

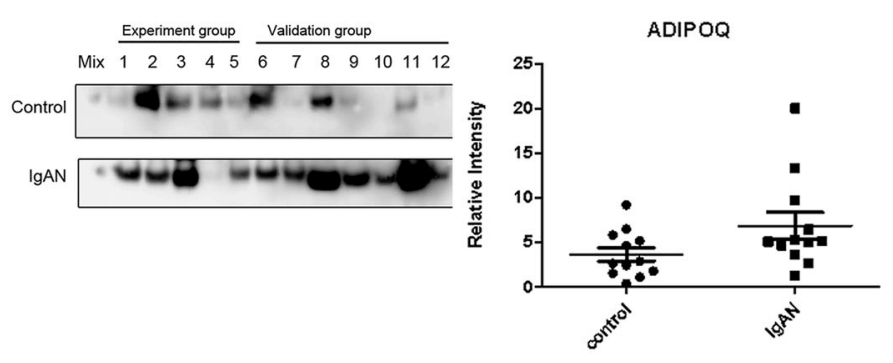

B

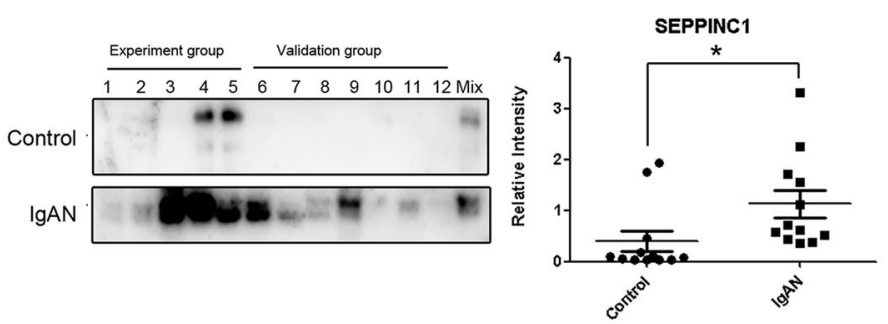

C

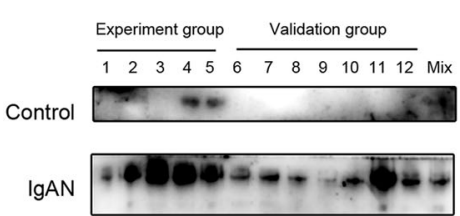

IgAN

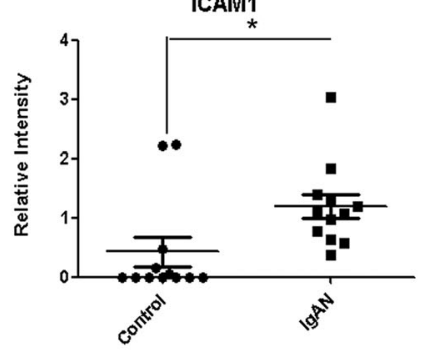

D
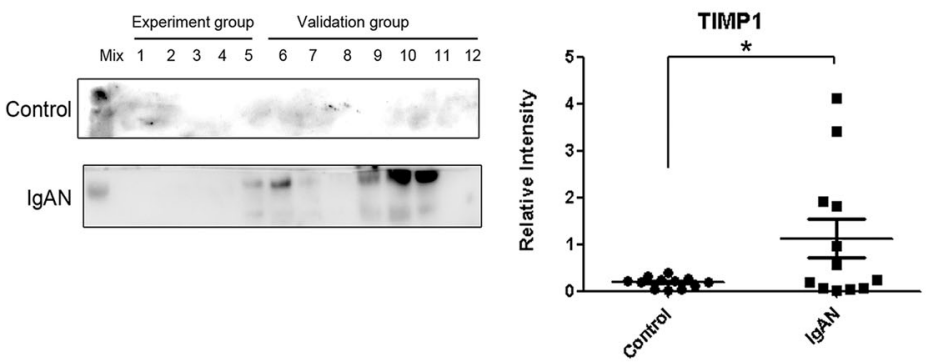
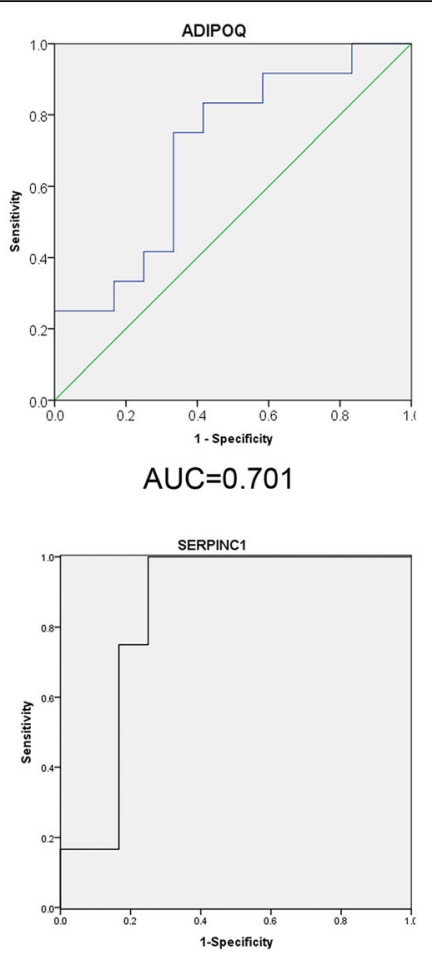

$\mathrm{AUC}=0.840$

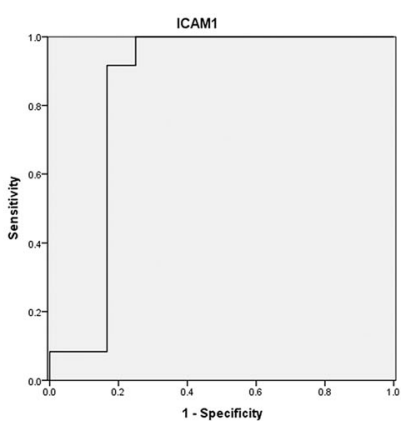

$A \cup C=0.840$

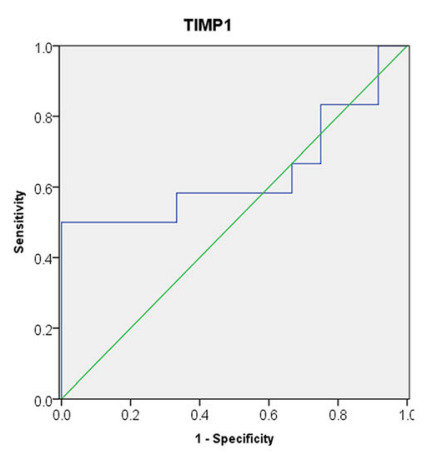

$\mathrm{AUC}=0.639$

Fig. 4 Western blot validation of four differentially expressed proteins. ADIPOQ (a), SERPINC1 (b), ICAM1 (c), and TIMP1 (d). ${ }^{*} p<0.05$; . ROC curves for Western blot validation of ADIPOQ (a), SERPINC1 (b), ICAM1 (c) and TIMP1 (d) in the IgAN group versus the control group are shown 
might be serum leaked proteins. However, the serum level of ADIPOQ also elevated in IgAN, the possible reason of the increase of ADIPOQ in urine needs further study (Additional file 4: Table S4).

In summary, the increase of ADIPOQ and SERPINC1 might originated from serum leakage, but TIMP1 and ICAM1 were not originated from serum leakage. Our study is a pilot research, the identification of biomarkers of IgAN were performed by comparison of IgAN patient and normal control. Though we compared the four biomarkers in the urine of IgAN with normal serum proteome to eliminate serum leaked proteins, it is difficult to completely exclude the serum leaked proteins. In our future study, to eliminate the influence of proteinuria, we will analyze the urinary proteomics in IgAN patients with different eGFR levels; meanwhile, we will further compare the urinary proteomics in IgAN with other related kidney diseases, which might identify the specific biomarkers of IgAN.

ICAM1 protein is a member of ligands for the leukocyte adhesion protein LFA-1 (integrin alpha-L/ beta-2). The tubular and interstitial expression of ICAM1 can be a marker of tubulointerstitial disturbance in IgAN [30]. Interstitial ICAM1 could be an adverse predictor of disease progression. Several studies have reported that urinary ICAM1 is increased in acute renal allograft rejection [31], chronic kidney disease (CKD) [32] and lupus nephritis [33, 34]. This study provides the first demonstration that ICAM1 is overrepresented in the urine of IgAN patients. ICAM1 serves as a kidney injury biomarker and increases in varies renal pathologies [30]. However, we do not know whether the urinary ICAM1 levels showed differences or not among different renal pathologies. In our future study, we will compare the ICAM1 levels between IgAN and other kidney dieseases, and we would estimate whether ICAM1 could be used as a successful specific urinary biomarkers for IgAN or not.

In IgAN, the deposition of extracellular proteins is increased in the mesangium. MMPs are the zincdependent endopeptidases of the matrix metalloproteinase families that degrade extracellular membrane (ECM) proteins, and TIMPs are the endogenous inhibitors of MMPs. Thus, the degree of ECM is controlled by the release of MMPs and their inhibition by TIMPs. Previous studies have shown that TIMP1 is overexpressed in the kidney tissue of diabetic nephropathy rats [29]. TIMP1 is elevated in post-liver transplantation renal recovery from acute kidney injury after liver transplantation [28]. This study, which includes validation through a Western blot analysis, provides the first demonstration of the upregulation of TIMP1 in the urine of Uygur patients with IgA nephropathy. We speculated that TIMP1 serves as a positive regulator of ECMs and plays a role in the production and deposition of extracellular proteins in the mesangium during the course of IgA nephropathy.

To better understand the pathophysiological changes in IgAN, we compared the differentially expressed proteins in IgAN identified in previous studies with those obtained in our study. A total of 166 differential proteins were identified in previous publications $[1,7-$ 11, 13, 39-43] (detailed data are shown in Additional file 5: Table S5). Only 47 of these overlapped with those found in our Uygur study (Fig. 5a). In addition, 36 of these 47 proteins showed consistent trends in this and previous studies.

An IPA analysis was performed to compare the differentially expressed proteins identified in previous studies and those identified in our Uygur study. The results of disease and functional analyses performed in previous studies and our study showed activation of the immune response in IgAN. More specifically, cell survival and viability, and phagocytosis were activated only in Uygur IgAN, as shown in our study (Fig. 5b). A pathway analysis revealed that the immune response and lipid metabolism-related pathway were enriched in IgAN in both previous studies and our Uygur study. This study found that the complement systems were more enriched in Uygur IgAN than previous studies (Fig. 5c). Therefore, our proteomic study reflects not only the common characteristics of IgAN that have been observed in previous studies but also special characteristics that are represented in Uygur IgAN but were not detected in previous studies. Because proteomic experiment of our study and previous studies are performed by different approaches and different laboratories, the differences results of IgAN urinary proteomics between our study and previous studies are still not very clear. It has been reported that IgAN shows marked variance between different ethnicities at the disease incidence and genome levels [14]. At the disease incidence level, IgAN is most common in Asians, is moderately prevalent in Europeans, and is rare in Africans [14]. At the genome level, Kiryluk et al. performed a GWAS to identify and confirm that five loci are significant contributors to the disease risk across this multi-ethnic cohort and used a genetic risk score to explain the worldwide patterns of disease prevalence [14]. Among the five loci, Chr.1q32 (CFHR3/R1 locus) encodes complement factor $\mathrm{H}$ $(\mathrm{CFH})$, complement factor $\mathrm{H}$-related protein 1 (CFHR1), CFHR2 and CFHR3 genes. Interestingly, $\mathrm{CFH}$, and CFHR2 were all significantly upregulated in the urine of Uygur IgAN patients. We speculated that the genome variation in IgAN would affect the proteome and we speculated that there are differences in the IgAN proteome and the pathological 
A

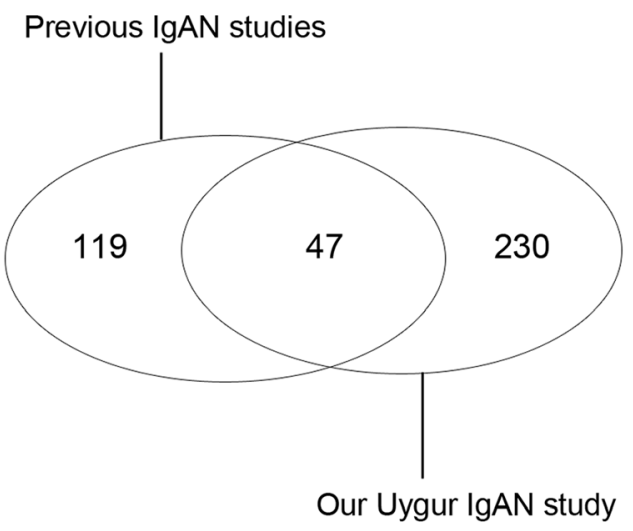

B

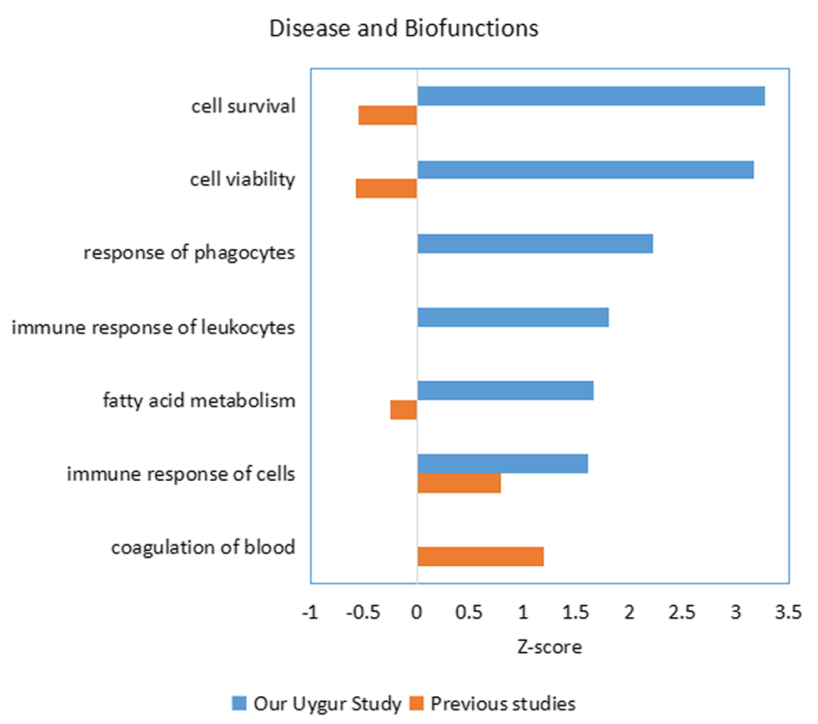

C

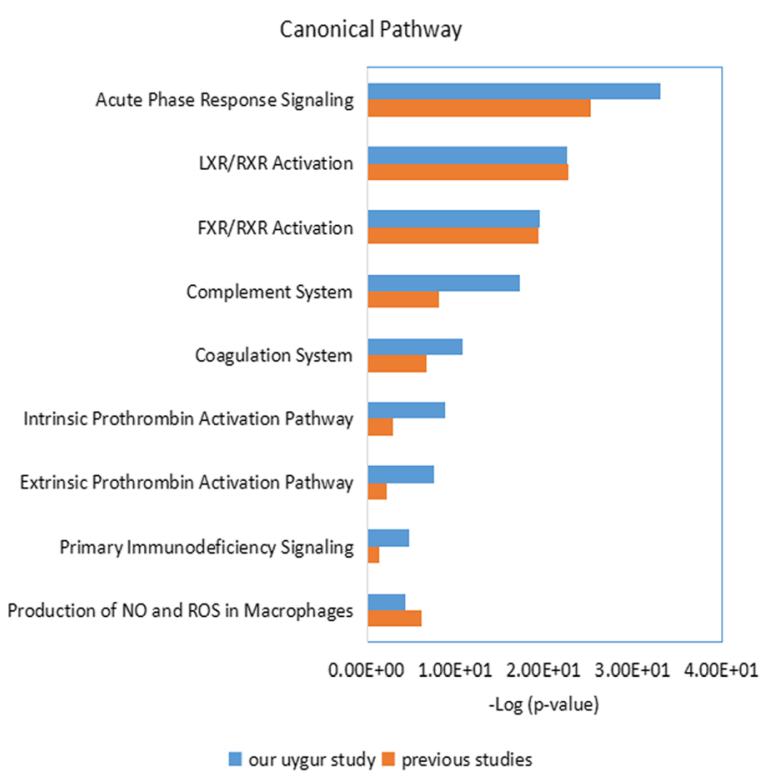

Fig. 5 Comparison of differentially expressed proteins in IgAN identified in our Uygur study and previous studies. a Venn diagram of differentially expressed proteins in IgAN obtained in our Uygur study and previous studies. $\mathbf{b}$ Disease and biofunction analysis of differentially expressed proteins in IgAN obtained in our Uygur study and previous studies. Z-score $>2$, significantly activated; Z-score $<-2$, significantly inhibited. c Top enriched canonical pathways in IgAN identified in this Uygur study and previous studies. - Log( $p$-value $)>1.5$, significantly enriched

mechanism between different ethnicities, and this hypothesis should be validated in a study that systematic compared between uygur population and other ethinities using the same approaches in the same laboratories.

\section{Conclusion}

In this study, we analyzed the differential urinary proteome of Uygur IgAN. A function analysis revealed that the immune response, cell survival, and complement system were activated in Uygur IgAN. Four candidate biomarkers were validated by Western blot analysis, and all of these were found to be upregulated in Uygur IgAN patients. Our study found that some of the differential proteins might be results of proteinuria. To identify the specific IgAN biomarkers, the proteinuia originated differential proteins should be eliminated in the future studies. Our findings would be helpful for a future exploration of the pathological mechanism of Uygur IgAN and will be helpful for the development of better treatments for IgAN patients of Uygur ethnicity.

\section{Additional files}

Additional file 1: Table S1. Clinical characteristics of Uygur normal controls and IgAN patients. (XLSX $13 \mathrm{~kb}$ )

Additional file 2: Table S2. Quantitative protein and peptide data. Quantitative values of peptides and proteins in the Uygur control and $\lg A N$ patients in runs 1 and 2 are shown. The sequence coverage of each protein in runs 1 and 2 are shown. (XLSX $421 \mathrm{~kb}$ ) 
Additional file 3: Table S3. Differentially expressed proteins in Uygur IgAN. (XLSX $19 \mathrm{~kb})$

Additional file 4: Table S4. Analysis the origin of the 4 candidate markers. (XLSX $10 \mathrm{~kb}$ )

Additional file 5: Table S5. Lists of differentially expressed proteins in IgAN obtained in previous studies. (XLS $66 \mathrm{~kb}$ )

\section{Abbreviations}

ACN: Acetonitrile; Alb: Serum albumin; AUC: Area-under-the-curve; BMI: Body mass index; BUN: Blood urea nitrogen; DBP: Diastolic blood pressure; DTT: Dithiothreitol; ECM: Extracellular membrane; eGFR: Estimated glomerular filtration rate; FASP: Filter-aided sample preparation; FBG: Fasting blood glucose; FDR: False discovery rate; GWAS: Genome-wide association study; HDL: High-density lipoprotein; IAM: Iodoacetamide; IPA: Ingenuity Pathway Analysis; iTRAQ: Isobaric tags for relative and absolute quantitation; LDL: Low-density lipoprotein; SBP: Systolic blood pressure; SCr: Serum creatinine; TG: Triglycerides; UAER: Urinary albumin excretion rates

\section{Acknowledgements}

Not applicable

\section{Funding}

This work was supported by the National Basic Research Program of China (No. 2013CB530805,2014CBA02005), National Key Research and Development Program of China (No. 2016 YFC 1306300), the Key Basic Research Program of the Ministry of Science and Technology of China (No. 2013FY114100), the National Natural Science Foundation of China (No. 30970650, 31200614 . $31400669,81371515,81170665$ and 81560121), the Science and Technology Yuanjiang Project of Xinjiang Uygur Autonomous Region (No. 2013911114), CAMS Innovation Fund for Medical Sciences (2017-12M-1-009) and Biologic Medicine Information Center of China, National Scientifc Data Sharing Platform for Population and Health, CAMS special basic research fund for central public research institutes (No. 2017PT310004).

\section{Availability of data and materials}

All data generated or analysed during this study are included in this published article.

\section{Authors' contributions}

Conceptualization: WS, HY, YG. Methodology: WS. Software: ZG. Validation: ZG. Formal analysis: ZG. Investigation: ZG, ZW, HS. Resources: CL, SY, R. Data curation: ZW. Writing (original draft preparation): ZG. Writing (review and editing): ZG, WS. Visualization: ZW. Supervision: WS, HY. Project administration: WS, HY, CL. Funding acquisition: WS, HY, CL, ZG. All authors read and approved the final manuscript.

\section{Ethics approval and consent to participate}

This study was approved by the Institutional Review Board of the Institute of Basic Medical Sciences and conformed to the principles outlined in the Declaration of Helsinki. Committee's reference number: 036-2014. Because our study used the remaining waste samples of the participants from clinical examination, and did not directly contact with the participants and would not regarded privacy of the participants, the Institutional Review Board of the Institute of Basic Medical Sciences waived the informed consents.

\section{Consent for publication}

Not applicable.

\section{Competing interests}

The authors declare that they have no competing interests.

\section{Publisher's Note}

Springer Nature remains neutral with regard to jurisdictional claims in published maps and institutional affiliations.

\section{Author details}

'Core Facility of Instrument, Institute of Basic Medicine, Chinese Academy of Medical Sciences, School of Basic Medicine, Peking Union Medical College, 5 Dong Dan San Tiao, Beijing, China. ${ }^{2}$ Nephrology department, The Xinjiang
Uygur Autonomous Region People's Hospital, 91 Tianchi Road, Urumqi, Xinjiang, China. ${ }^{3}$ Graduate School, Xinjiang Medical University, 393 Xinyi Road, Urumqi, Xinjiang, China.

Received: 1 October 2017 Accepted: 15 November 2018

Published online: 14 December 2018

\section{References}

1. Rocchetti MT, Centra M, Papale M, Bortone G, Palermo C, et al. Urine protein profile of IgA nephropathy patients may predict the response to ACE-inhibitor therapy. Proteomics. 2008:8:206-16.

2. Donadio JV, Grande JP. IgA nephropathy. N Engl J Med. 2002;347:738-48.

3. Appel GB, Waldman M. The IgA nephropathy treatment dilemma. Kidney Int. 2006;69:1939-44

4. Wu J, Wang N, Wang J, Xie Y, Li Y, et al. Identification of a uromodulin fragment for diagnosis of IgA nephropathy. Rapid Commun Mass Spectrom. 2010;24:1971-8

5. Eiro M, Katoh T, Watanabe T. Risk factors for bleeding complications in percutaneous renal biopsy. Clin Exp Nephrol. 2005;9:40-5.

6. Guo Z, Liu X, Li M, Shao C, Tao J, et al. Differential urinary glycoproteome analysis of type 2 diabetic nephropathy using 2D-LC-MS/MS and iTRAQ quantification. J Transl Med. 2015;13:371.

7. Park MR, Wang EH, Jin DC, Cha JH, Lee KH, et al. Establishment of a 2-D human urinary proteomic map in IgA nephropathy. Proteomics. 2006;6:1066-76.

8. Surin B, Sachon E, Rougier JP, Steverlynck C, Garreau C, et al. LG3 fragment of endorepellin is a possible biomarker of severity in IgA nephropathy. Proteomics. 2013;13:142-52.

9. Sui W, Cui Z, Zhang R, Xue W, Ou M, et al. Comparative proteomic analysis of renal tissue in IgA nephropathy with iTRAQ quantitative proteomics. Biomed Rep. 2014;2:793-8.

10. Mucha K, Bakun M, Jazwiec R, Dadlez M, Florczak M, et al. Complement components, proteolysisrelated, and cell communicationrelated proteins detected in urine proteomics are associated with IgA nephropathy. Pol Arch Med Wewn. 2014;124:380-6.

11. Zhao S, Li R, Cai X, Chen W, Li Q, et al. The application of SILAC mouse in human body fluid proteomics analysis reveals protein patterns associated with IgA nephropathy. Evid Based Complement Alternat Med. 2013;2013:275390

12. Moon PG, Lee JE, You S, Kim TK, Cho JH, et al. Proteomic analysis of urinary exosomes from patients of early IgA nephropathy and thin basement membrane nephropathy. Proteomics. 2011;11:2459-75.

13. Kalantari S, Rutishauser D, Samavat S, Nafar M, Mahmudieh L, et al. Urinary prognostic biomarkers and classification of $\lg A$ nephropathy by high resolution mass spectrometry coupled with liquid chromatography. PLoS One. 2013;8:e80830.

14. Kiryluk K, Li Y, Sanna-Cherchi S, Rohanizadegan M, Suzuki H, et al. Geographic differences in genetic susceptibility to IgA nephropathy: GWAS replication study and geospatial risk analysis. PLoS Genet. 2012:8:e1002765.

15. Li WL, Lu C. Association between C1GALT1 variants and genetic susceptibility to IgA nephropathy in Uygur. Genet Mol Res. 2015;14:5327-33.

16. Yue H, Zhou J, Adila, He W, Qiao LP, et al. Analysis of pathological and clinical data of renal biopsy in 237 Uygur patients in Xinjiang. Xinjiang Medical Journal. 2006;36:1-3.

17. Zhang $L$, Wang F, Wang $L$, et al. Prevalence of chronic kidney disease in China: a cross-sectional survey. Lancet. 2012;379(9818):815-22.

18. Xu X, Ning Y, Shang W, et al. Analysis of 4931 renal biopsy data in Central China from 1994 to 2014. Ren Fail. 2016:38(7):1021-30.

19. Zhou FD, Zhao MH, Zou WZ, et al. The changing spectrum of primary glomerular diseases within 15 years: a survey of 3331 patients in a single Chinese Centre. Nephrol Dial Transplant. 2009;24(3):870-6.

20. Zhang $X, S L$, Tang $L$, et al. Analysis of pathological data of renal biopsy at one single center in China from 1987 to 2012. Chin Med J. 2014;127(9):1715-20.

21. Lu C, Zhu K, Zhao H, Ji J, Mu G, et al. Correlation between TCRCalpha -560 $\mathrm{C} / \mathrm{T}$ polymorphism and the clinical presentation of Uygur IgA nephropathy patients in XinJiang. Asian Pac J Allergy Immunol. 2011;29:236-9.

22. Barratt J, Feehally J. IgA nephropathy. J Am Soc Nephrol. 2005;16:2088-97.

23. Moriyama T, Oshima Y, Tanaka K, Iwasaki C, Ochi A, et al. Statins stabilize the renal function of IgA nephropathy. Ren Fail. 2014:36:356-60.

24. Fukui $H$, Taniguchi A, Sakamoto S, Kawahara S, Matsunaga T, et al. Antithrombin III in children with various renal diseases. Pediatr Nephrol. 1989:3:144-8 
25. Ohashi K, Iwatani H, Kihara S, Nakagawa Y, Komura N, et al. Exacerbation of albuminuria and renal fibrosis in subtotal renal ablation model of adiponectin-knockout mice. Arterioscler Thromb Vasc Biol. 2007;27:1910-7.

26. Inoue $T$, Sugiyama $H$, Kitagawa $M$, Takiue $K$, Morinaga $H$, et al. Suppression of adiponectin by aberrantly glycosylated lgA1 in glomerular mesangial cells in vitro and in vivo. PLoS One. 2012;7:e33965.

27. Jin X, Chen J, Hu Z, Chan L, Wang Y. Genetic deficiency of adiponectin protects against acute kidney injury. Kidney Int. 2013;83:604-14.

28. Levitsky J, Baker TB, Jie C, Ahya S, Levin M, et al. Plasma protein biomarkers enhance the clinical prediction of kidney injury recovery in patients undergoing liver transplantation. Hepatology. 2014;60:2017-26.

29. Dong J, Liu X, Liu S, Li M, Xu Y, Cui B. Effects of calcium dobesilate on glomerulus TIMP1 and collagen IV of diabetic rats. J Huazhong Univ Sci Technolog Med Sci. 2005;25(4):416-8 426.

30. Arrizabalaga P, Sole M, Abellana R, de las Cuevas X, Soler J, et al. Tubular and interstitial expression of ICAM-1 as a marker of renal injury in IgA nephropathy. Am J Nephrol. 2003;23:121-8.

31. Roberti I, Reisman L. Serial evaluation of cell surface markers for immune activation after acute renal allograft rejection by urine flow cytometry-correlation with clinical outcome. Transplantation. 2001;71:1317-20.

32. Liu BC, Zhang L, Lv LL, Wang YL, Liu DG, et al. Application of antibody array technology in the analysis of urinary cytokine profiles in patients with chronic kidney disease. Am J Nephrol. 2006;26:483-90.

33. Abd-Elkareem Ml, Al Tamimy HM, Khamis OA, Abdellatif SS, Hussein MR. Increased urinary levels of the leukocyte adhesion molecules ICAM-1 and VCAM-1 in human lupus nephritis with advanced renal histological changes: preliminary findings. Clin Exp Nephrol. 2010;14:548-57.

34. Guan J, Wang G, Tam LS, Kwan BC, Li EK, et al. Urinary sediment ICAM-1 level in lupus nephritis. Lupus. 2012;21:1190-5.

35. Shimotomai T, Kakei M, Narita T, Koshimura J, Hosoba M, et al. Enhanced urinary adiponectin excretion in IgA-nephropathy patients with proteinuria. Ren Fail. 2005;27:323-8.

36. Nagaraj N, Mann M. Quantitative analysis of the intra- and inter-individual variability of the normal urinary proteome. J Proteome Res. 2011;10:637-45.

37. Guo Z, Zhang Y, Zou L, Wang D, Shao C, Wang Y, Sun W, Zhang L. A proteomic analysis of individual and gender variations in Normal human urine and cerebrospinal fluid using iTRAQ quantification. PLoS One. 2015; 10(7):e0133270.

38. Geyer PE, Kulak NA, Pichler G, Holdt LM, Teupser D, Mann M. Plasma proteome profiling to assess human health and disease. Cell Syst. 2016;2(3): 185-95.

39. Jang HR, Kim SM, Lee YJ, Lee JE, Huh W, Kim DJ, Oh HY, Kim YG. The origin and the clinical significance of urinary angiotensinogen in proteinuric $\lg \mathrm{A}$ nephropathy patients. Ann Med. 2012:44(5):448-57.

40. Wasilewska A, Zoch-Zwierz W, Taranta-Janusz K, Kołodziejczyk Z. Urinary monocyte chemoattractant protein-1 excretion in children with glomerular proteinuria. Scand J Urol Nephrol. 2011;45(1):52-9.

41. Longhi A, Ferrari S, Tamburini A, Luksch R, Fagioli F, Bacci G, Ferrari C. Late effects of chemotherapy and radiotherapy in osteosarcoma and Ewing sarcoma patients: the Italian sarcoma group experience (1983-2006). Cancer. 2012;118(20):5050-9

42. Rocchetti MT, Papale M, d'Apollo AM, Suriano IV, Di Palma AM, Vocino G, et al. Association of urinary laminin G-like 3 and free K light chains with disease activity and histological injury in IgA nephropathy. Clin J Am Soc Nephrol. 2013;8(7):1115-25.

43. Shi B, Wang L, Mou S, Zhang M, Wang Q, Qi C, et al. Identification of mannose-binding lectin as a mechanism in progressive immunoglobulin a nephropathy. Int J Clin Exp Pathol. 2015;8(2):1889-99.

\section{Ready to submit your research? Choose BMC and benefit from:}

- fast, convenient online submission

- thorough peer review by experienced researchers in your field

- rapid publication on acceptance

- support for research data, including large and complex data types

- gold Open Access which fosters wider collaboration and increased citations

- maximum visibility for your research: over $100 \mathrm{M}$ website views per year

At BMC, research is always in progress.

Learn more biomedcentral.com/submissions 